\title{
Heartbeat: diagnosis and management of pericardial disease
}

doi:10.1136/heartjnl-2021-319144

Catherine M Otto

Pericardial disease is a common clinical problem yet treatment remains empiric with few clinical trials evaluating medical therapy. Klein and colleagues ${ }^{1}$ hypothesised that rilonacept, an inhibitor of both interleukin-1 alpha (IL-1 $\alpha$ ) and IL-1 $\beta$, would reduce pain and signs of inflammation, while improving health-related quality of life in patients with recurrent pericarditis. Of the 25 patients enrolled in this phase II clinical trial, pericarditis pain was reduced from 4.5 to 0.7 on an 11-point pain scale and $\mathrm{C}$ reactive protein levels decreased from a mean of $4.62 \mathrm{mg} /$ $\mathrm{dL}$ at baseline to $0.38 \mathrm{mg} / \mathrm{dL}$ at a median time of 9 days after treatment, with most being able to discontinue corticosteroid therapy (figure 1).

As Imazio concludes in an editorial ${ }^{2}$ 'anti-IL-1 agents appear to be the major advance in medical therapy of recurrent pericarditis in the last 5 years (figure 2). If efficacy and safety will be confirmed, these drugs may represent a paradigm shift in the treatment of recurrent pericarditis, allowing a more targeted and personalised therapy for patients showing an inflammatory phenotype (eg, fever and/or C-reactive elevation at each recurrence'.

Cardiac compression due to pericardial effusion (tamponade physiology) or pericardial scarring and fibrosis (pericardial constriction) are the ends of a spectrum of pericardial compressive syndromes. Effusive-constrictive pericarditis is less common but occurs when there is 'concurrence of a tense pericardial effusion and constriction of the heart by the visceral pericardium' as reviewed by Janus and $\mathrm{Hoit}^{3}$ in this issue of Heart. Effusiveconstrictive pericarditis may be related to malignancy, irradiation therapy, prior cardiac surgery or other causes. Diagnosis and management are challenging so readers will find this comprehensive article helpful in diagnosis and management of patients with fluid overload or low cardiac output that might be due to pericardial disease (figure 3).

Inequities in clinical care for cardiovascular disease (CVD) contribute to higher rates of adverse outcomes. In this issue of Heart, Pinho-Gomes and colleagues ${ }^{4}$

Correspondence to Professor Catherine M Otto, Division of Cardiology, University of Washington, Seattle, WA 98195, USA; cmotto@uw.edu

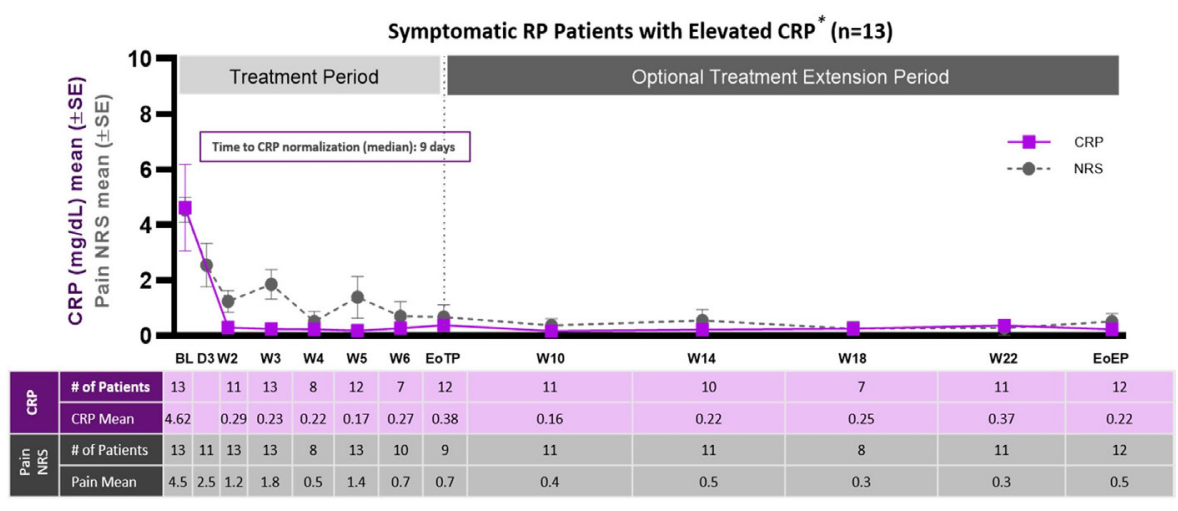

Figure 1 NRS scores (pain) and CRP levels in symptomatic patients with elevated CRP. CRP, C reactive protein; NRS, Numeric Rating Scale; RP, recurrent pericarditis.

investigated sex differences in the prevalence, treatment and control of major CVD risk factors in the UK based on data from the Health Survey for England (figure 4). Overall, men had a higher number of CVD risk factors (smoking, hypertension, overweight and dyslipidaemia) than women. Between 2012 and 2017, there was an increase in the number of individuals with none of these risk factors, from $32 \%$ to
$36 \%$ in women and from $28 \%$ to $29 \%$ in men. Over time, greater numbers of patients of both sexes received treatment and achieved control of hypertension and diabetes, although control still remained suboptimal for both risk factors (hypertension 51\%, diabetes 20\%). Dyslipidaemia treatment rates were poor in both sexes but were lower in women compared with men $(21 \%$ vs $28 \%)$. The authors conclude

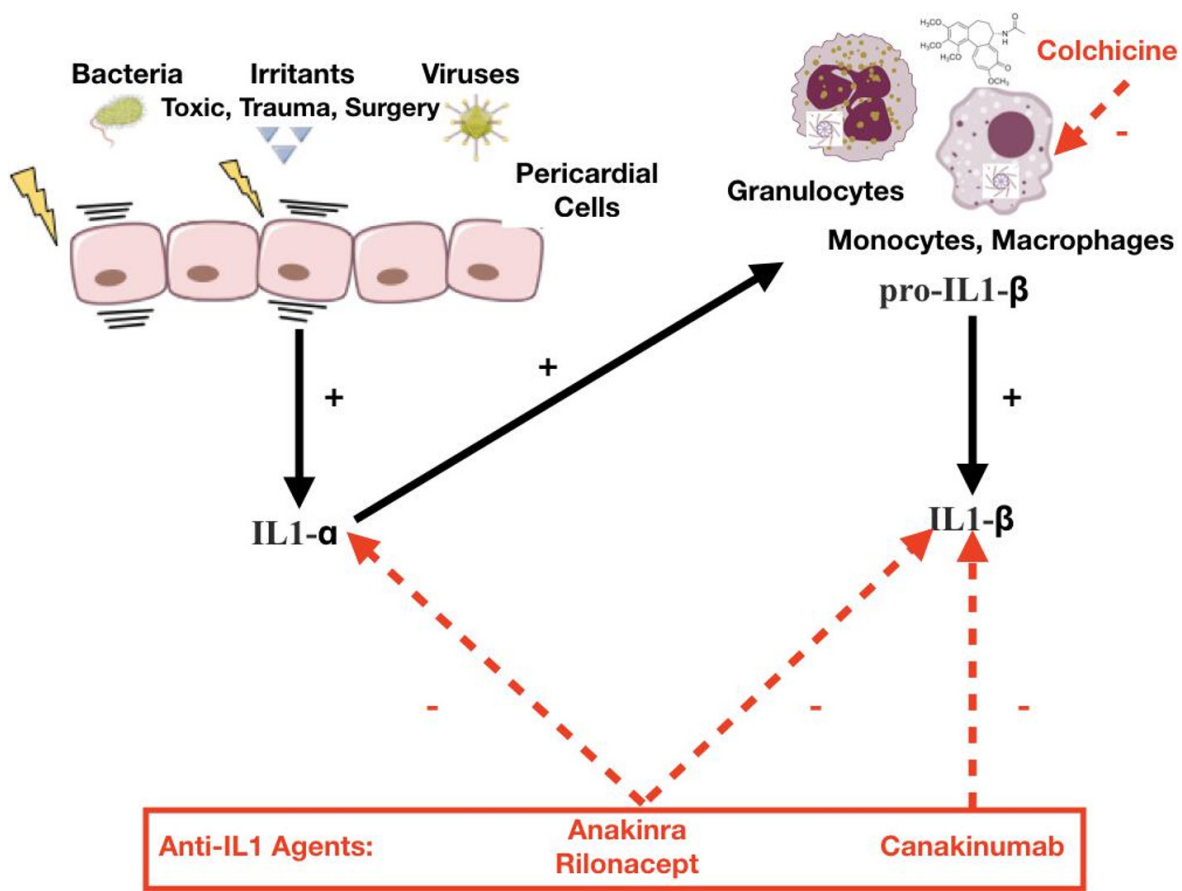

Figure 2 Pathophysiology of pericardial inflammation and targets for pharmacological intervention: inflamed pericardial cells release IL-1-alpha that further triggers release of IL-1-beta from inflammatory cells. Colchicine inhibits inflammatory cells and the inflammasome, while antiIL-1 agents block IL-1 alpha and/or beta. IL, interleukin. 


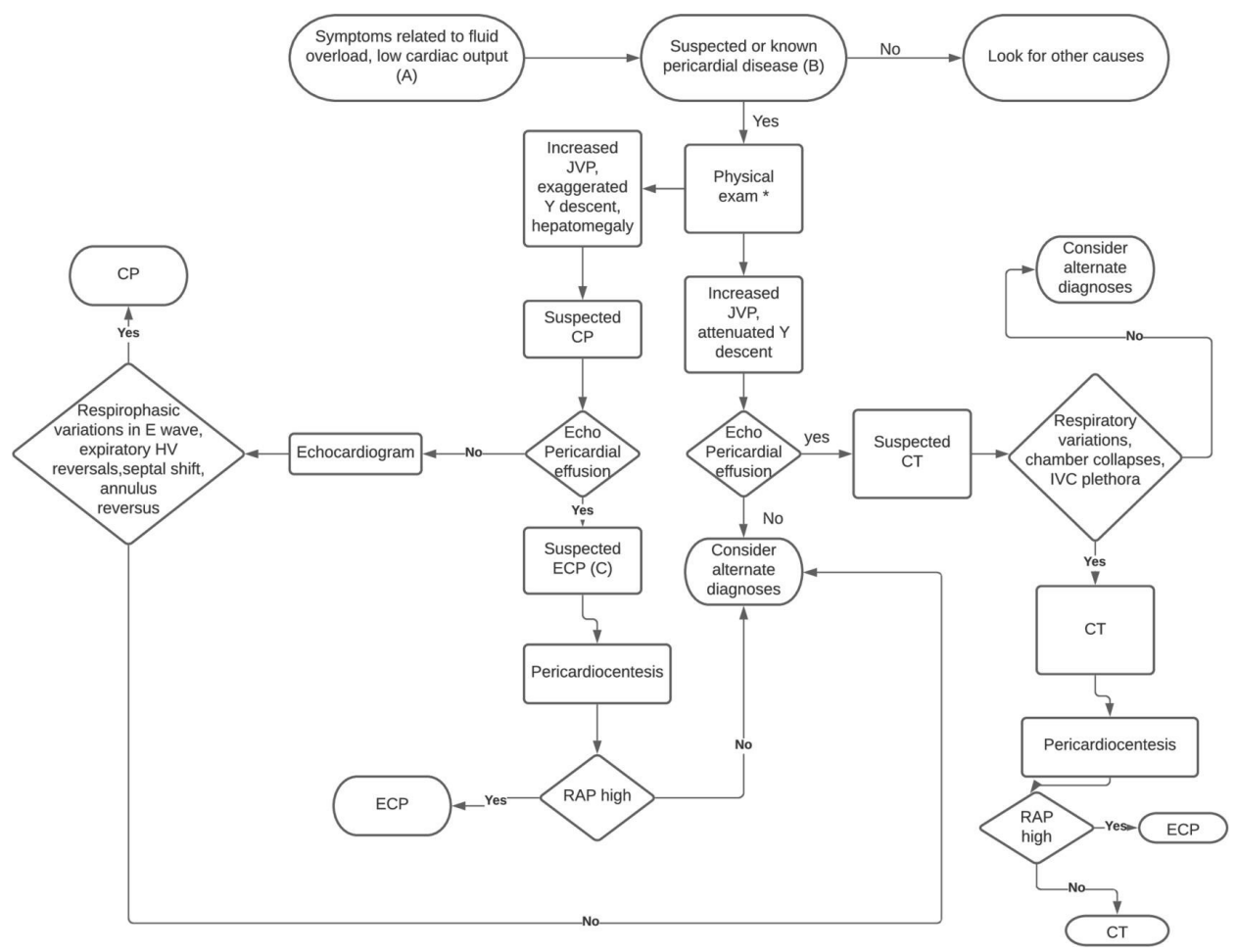

Figure 3 Flowchart illustrating an approach to the diagnosis of the pericardial compressive syndromes using clinical examination and echocardiography. Symptoms related to fluid overload and low cardiac output state (A) include oedema, anasarca, fatigue and dyspnoea in a patient with suspected or known pericardial disease (B), such as acute pericarditis, postinjury pericarditis (including iatrogenic), malignancy and connectivetissue and renal disease. Echocardiographic findings of ECP $(C)$ include elevated medial mitral E', respirophasic septal shift, inferior vena cava plethora, rapid transmitral E velocity and HV expiratory reversals. *In lieu of an examination of the JVP, right heart catheterisation is used to measure RAP. CT, cardiac tamponade; CP, constrictive pericarditis; ECP, effusive-constrictive pericarditis; HV, hepatic vein; IVC, inferior vena cava; JVP, jugular venous pressure; RAP, right atrial pressure.

that 'A combination of public health policy and individually tailored interventions is required to further reduce the burden of cardiovascular disease in England.'

Also in this issue of Heart is a short summary of the key changes in the European Society of Cardiology 2020
Guidelines for management of acute coronary syndromes without ST-elevation. ${ }^{5}$ A few of the major points are use of 0 and 1 hours rapid rule-out protocols, a preference for prasugrel over ticagrelor in patients undergoing invasive intervention, delayed angiography after resuscitated out-of-hospital cardiac arrest in haemodynamically stable patients and consideration of complete revascularisation in patients with multivessel disease who are not in cardiogenic shock.

The unfolding story of the interaction between the COVID-19 pandemic and
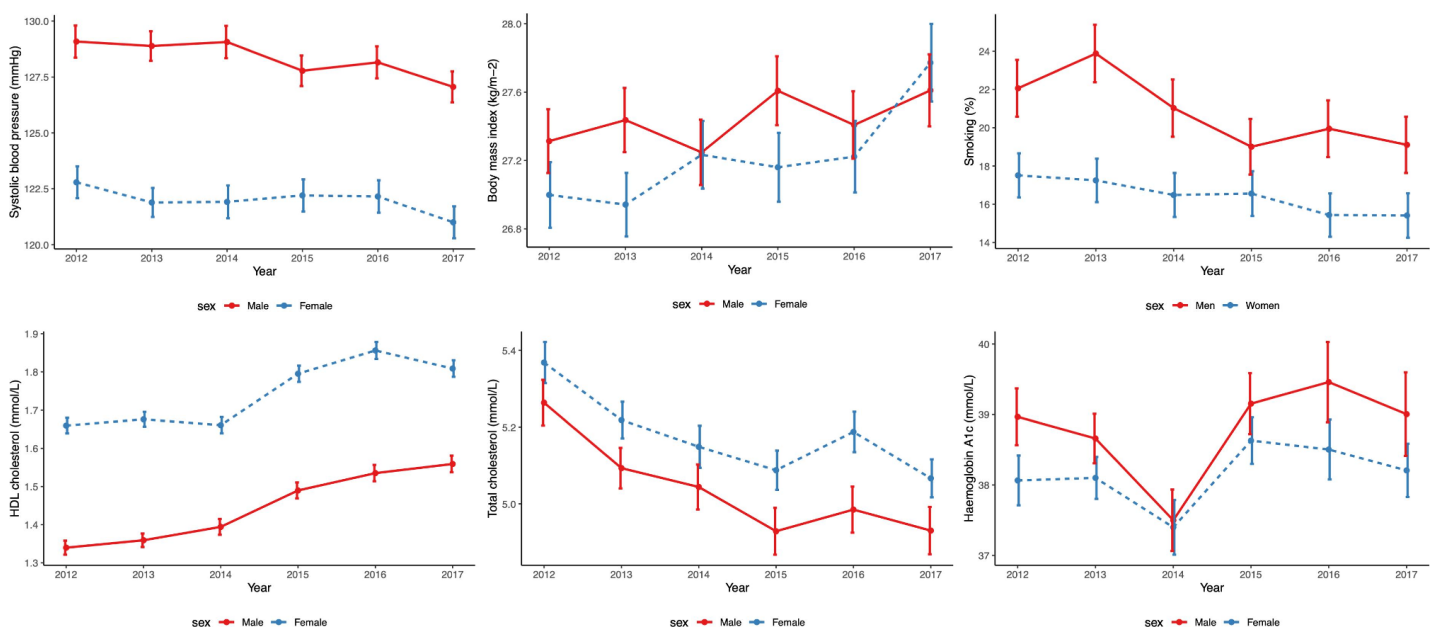

Figure 4 Trends in cardiovascular risk factors by sex between 2012 and 2017. All estimates are age-standardised to the English standard population in 2017. Error bars indicate $95 \% \mathrm{Cls}$. A total of 25254 women and 24161 men participated in the surveys between 2012 and 2017 . The number of participants for each year was 4270, 4553, 4165, 4107, 4082 and 4077 for women and 4081, 4317, 3987, 3927, 3929 and 3920 for men from 2012 to 2017, respectively. BMI, body mass index; BP, blood pressure; HbA1c, haemoglobin A1 c; HDL, high-density lipoprotein. 


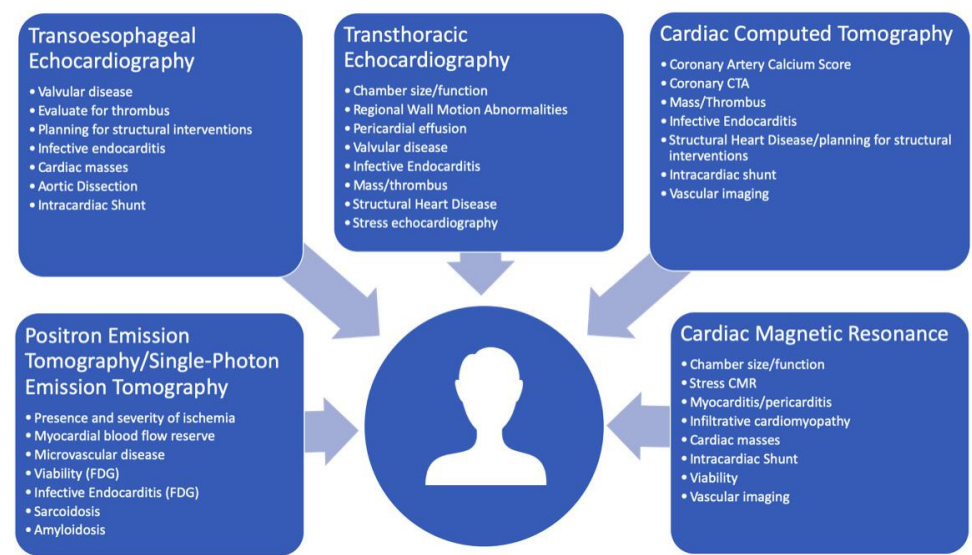

Figure 5 Patient-centred imaging and common indications for each modality. CMR, cardiovascular magnetic resonance; CTA, CT angiography; FDG, fluorodeoxyglucose.

cardiovascular mortality is addressed in a systematic review and meta-analysis showing that despite reduced rates of admission for ST-elevation myocardial infarction (STEMI) during the COVID-19 pandemic, hospital mortality was unchanged. ${ }^{6}$ Danchin and Marijon ${ }^{7}$ express the concern that although this apparent decrease in STEMI might be due to an actual decrease in event rates, it also is possible that if the decrease was primarily due to fear of getting admitted in patients actually having had an AMI, we should observe an unusually increased rate in late complications potentially related to larger myocardial infarctions caused by the lack of appropriate management at the acute stage (eg, congestive heart failure or ventricular arrhythmias).' The recent study by Wu and colleagues ${ }^{8}$ and the accompanying editorial ${ }^{9}$ both support the latter possibility-patients are failing to seek medical care for acute cardiovascular events during the COVID-19 pandemic resulting in long-term adverse consequences of delayed therapy.
The Education in Heart article ${ }^{10}$ in this issue defines multimodality imaging as 'using a patient-centred approach to select the best available imaging test when evaluating patients with known or suspected cardiovascular disease' (figure 5). The article then focuses on multimodality imaging for ischaemic heart disease; discussing the strengths and limitation of exercise treadmill testing, coronary CT angiography, exercise or pharmacological single-photon emission (SPECT) , or positron emission tomographic (PET) myocardial perfusion imaging, stress cardiovascular magnetic resonance or stress echocardiography. The authors point out that 'Effective delivery of multimodality imaging requires collaboration between imagers and referring physicians, as well as training of dedicated future multimodality imaging experts.'

Funding The authors have not declared a specific grant for this research from any funding agency in the public, commercial or not-for-profit sectors.

Competing interests None declared.
Patient and public involvement Patients and/or the public were not involved in the design, or conduct, or reporting, or dissemination plans of this research.

Patient consent for publication Not required.

Provenance and peer review Commissioned; internally peer reviewed.

(C) Author(s) (or their employer(s)) 2021. No commercial re-use. See rights and permissions. Published by BMJ.

\section{Check for updates}

To cite Otto CM. Heart 2021;107:433-435.

Heart 2021:107:433-435.

doi:10.1136/heartjnl-2021-319144

\section{ORCID iD}

Catherine M Otto http://orcid.org/0000-0002-05279392

\section{REFERENCES}

1 Klein AL, Lin D, Cremer PC. Efficacy and safety of rilonacept for recurrent pericarditis: results from a phase II clinical trial. Heart 2021;107:488-496.

2 Imazio M. Anti-IL-1 agents: a paradigm shift in medical therapy for recurrent pericarditis? Heart 2021;107:438-40.

3 Janus SE, Hoit BD. Effusive-Constrictive pericarditis in the spectrum of pericardial compressive syndromes. Heart 2021:107:450-5.

4 Pinho-Gomes AC, Peters SAE, Thomson B. Sex differences in prevalence, treatment and control of cardiovascular risk factors in England. Heart 2021;107:462-7.

5 Meah MN, Mills NL, Adamson PD. The 2020 European Society of cardiology non-ST-segment elevation acute coronary syndromes guideline: the good, the bad and the ugly. Heart 2021:107:444-6.

6 Rattka M, Dreyhaupt J, Winsauer C. Effect of the COVID-19 pandemic on mortality of patients with STEMI: a systematic review and meta-analysis. Heart 2021;107:482-7.

7 Danchin N, Marijon E. COVID-19 pandemic: preventing Hospital myocardial infarction admissions or preventing acute myocardial infarction altogether? Heart 2021;107:436-7.

8 Wu J, Mamas MA, Mohamed MO, et al. Place and causes of acute cardiovascular mortality during the COVID-19 pandemic. Heart 2021;107:113-9.

9 Singh T, Newby DE. Is the fear of disease worse than the disease itself? Heart 2021;107:91-2.

10 Madamanchi C, Di Carli MF, Blankstein R. What is multimodality cardiovascular imaging and how can it be delivered? Heart 2021;107:503-8. 